\title{
Antoine Bevort, Annette Jobert, Sociologie du travail : les relations professionnelles
}

\section{Christian Dufour}

\section{Q OpenEdition}

1 Journals

Édition électronique

URL : http://journals.openedition.org/travailemploi/1807

DOI : 10.4000/travailemploi.1807

ISSN : 1775-416X

Éditeur

DARES - Ministère du Travail

\section{Édition imprimée}

Date de publication : 5 décembre 2008

Pagination : 83-84

ISSN : 0224-4365

\section{Référence électronique}

Christian Dufour, "Antoine Bevort, Annette Jobert, Sociologie du travail : les relations professionnelles », Travail et Emploi [En ligne], 116 | octobre-décembre 2008, mis en ligne le 05 novembre 2010, consulté le 22 septembre 2020. URL : http://journals.openedition.org/travailemploi/1807 ; DOI : https://doi.org/ 10.4000/travailemploi. 1807 


\section{Sociologie du travail: les relations professionnelles Antoine Bevort et Annette Jobert}

\author{
Armand Colin, 2008, 268 pages
}

\section{Lu par Christian Dufour(1)}

Les auteurs de cet ouvrage, qui paraît dans une collection à vocation pédagogique, se proposent de livrer "une analyse générale des relations professionnelles françaises, dans une perspective historique et comparative. » (pp. 17). L'ambition pratique - «la connaissance des dimensions concrètes des relations professionnelles (stratégies et pratique des acteurs, fonctionnement de la négociation collective, nature des conflits) »-s'appuie sur un principe théorique qui voit dans la production de règles «l'enjeu central des relations professionnelles». Pour concilier pédagogie et réflexion sur une discipline et ses objets dans le contexte français, le titre associe deux disciplines sécantes, la sociologie du travail et les relations professionnelles. L'objectif pédagogique passe par un découpage rigoureux de l'ouvrage.

L'introduction générale retrace les étapes essentielles de la construction des relations professionnelles, en tant qu'objet social et en tant que discipline. Les sources nord-américaines et anglaises sont privilégiées. Le lecteur est ainsi conduit à s'interroger sur l'originalité de la situation française qui méconnaît cette discipline, tant du point de vue des raisons historiques de cette originalité que des raisons plus académiques. L'introduction offre aussi une exposition concentrée des principes méthodologiques et théoriques qui guident les auteurs.

\section{" Acteurs et institutions"}

La première partie et ses cinq chapitres permettent d'identifier les éléments principaux à travers lesquels s'exercent concrètement les relations professionnelles. Trois chapitres sont réservés aux acteurs («les syndicats», «les patronats», «l'État») et deux aux institutions ( $\ll$ les institutions représentatives du personnel (IRP)», «le paritarisme»). Chaque chapitre - à l'exception de celui sur les IRP - s'attache d'abord à une présentation historique de son objet particulier. Une chronologie vient soutenir l'analyse de la formation du syndicalisme français (pp. 28-29), et celle du paritarisme (pp. 120-121). Chaque chapitre comporte aussi une série de références à des situations nationales non françaises, pour proposer une mise en perspective comparative (c'est le cas par exemple de l'opposition entre système dual-représentation assurée simultanément par les voies syndicale et élective - et système moniste - représentation par la voie syndi-

(1) IRES. cale seule). Chaque chapitre se clôt fort utilement par une conclusion qui offre un espace de réflexion problématique sur l'objet de cette partie.

Conformément à l'objectif de l'ouvrage, on trouve dans ces chapitres l'essentiel des connaissances nécessaires à une première approche de ces acteurs et de ces institutions. Les auteurs vont à l'essentiel pour présenter les différentes formes de syndicalisme et les structures du syndicalisme français. Le chapitre sur le patronat, par la description de ses multiples modes d'intervention, met bien en relief que «la représentation et l'action patronale empruntent une logique spécifique différente de celle des syndicats » (p. 69). Celui sur les IRP insiste sur les différences entre secteur privé et secteur public tout en présentant les découpages institutionnels. Les rôles réciproques, complémentaires ou contradictoires, de l'État et du paritarisme sont clairement présentés.

Cette partie a en outre l'intérêt d'ouvrir la discussion sur plusieurs points. En ce qui concerne les syndicats d'abord, on peut se demander si l'affirmation que la "première spécificité du cas français»" est «dans le secret qui entoure le nombre de syndiqués» (p. 22) ne doit pas être nuancée. D'autres traits ne le distinguent-ils pas plus fortement, comme le montrent les pages suivantes de l'ouvrage? D'autant que la transparence prêtée à d'autres pays en la matière n'est pas avérée. De même, en ce qui concerne l'État, le fait que, en Allemagne, «en pratique, sinon en droit, les accords collectifs s'appliquent à tous les salariés du secteur considéré et non aux seuls membres du syndicat» (p. 89) devrait être réinterrogé par les recherches récentes. C'est l'appartenance de l'employeur à une organisation qui est décisive comme dans bon nombre de pays (Suède...), pas celle des salariés. Et beaucoup d'employeurs quittent leurs organisations pour éviter d'appliquer les conventions collectives. Dans le même pays, les réformes Hartz, qui ont bouleversé le marché du travail, ont été prises sans l'aval des syndicats, par le gouvernement Schröder(2). Désormais, les relations entre le parti social-démocrate et les syndicats sont profondément bouleversées. Il n'est plus vrai que «le DGB...ne peut être tenu à l'écart des grands arbitrages socio-économiques du pays particulièrement quand les socialistes sont au pouvoir» (ibidem).

\section{Conflits, négociations et nouvelles régulations}

La deuxième partie intitulée Conflits, négociations et nouvelles régulations s'ouvre sur un chapitre - «De la confrontation sociale à la fracture sociale» (pp.143-166) - qui inscrit les relations professionnelles dans le cadre sociétal français de long terme. Il en révéle les structures spécifiques, la «tradition» qui contribue à rendre le système

(2) Cf. C. Logeay et K. Rietzler (2008) «Réformes du marché du travail en Allemagne - aucun effet sur l'emploi et aggravation des déséquilibres en Europe " La Revue de l'IRES, $n^{\circ} 56,2008 / 1$, pp. 3-39. 
chaotique. Ce chapitre dresse un tableau historique dense des conditions - souvent agitées - dans lesquelles se forme le système de relations professionnelles français. Le milieu des années 1930, la Libération, le tournant de la fin des années 1960, les premiers effets de la crise économique puis le changement de millénaire marquent les grandes étapes de l'évolution de la confrontation sociale vers la fracture sociale. Cette partie se poursuit par des chapitres sur "les conflits sociaux», "la négociation collective», «L'Europe des relations professionnelles» et «Les transformations des régulations professionnelles», ce dernier chapitre servant de conclusion à l'ouvrage. À travers ces chapitres, les auteurs entrent plus directement dans les questions qui traversent les pratiques et les stratégies des acteurs des relations professionnelles. Les transformations du salariat, la formation d'une l'Europe sociale «qui marque le pas» mais se dote d'instruments nouveaux (dialogue social, comités d'entreprise européens...), les exigences de la mondialisation à l'égard des «transformations des régulations professionnelles» sont passées en revue.

Dans cette partie cependant, les trois premiers chapitres ne font pas référence à la méthode comparative et parfois historique (comme dans le cas du chapitre sur la négociation). Le chapitre qui l'ouvre, sur «la confrontation sociale...», fort bienvenu, est placé un peu tard dans l'ouvrage dont il transforme la tonalité. En début de première partie, il en aurait renforcé la problématisation, et rendu la description des acteurs et des institutions plus vivante. Un déplacement du chapitre sur le paritarisme aurait permis de sauvegarder les principes d'équilibre entre parties. La conclusion de ce sixième chapitre comporte en effet une proposition forte des auteurs. Pour ces derniers, «le modèle français affronte depuis bientôt vingt années une crise d'efficacité et de légitimité indiscutable... La forte perte de crédit du syndicalisme signe sa délégitimation...» (p. 165). Cette perspective, quoique énoncée tardivement, semble en fait structurer l'ouvrage. Une recherche lexicographique pourrait dénombrer les appels des auteurs à la question de la légitimité - fréquente - et les comparer aux appels aux règles - rares.

Telle nous semble être en fait la stimulante question de l'ouvrage, celle de la légitimité des acteurs, plus que celle des règles qu'ils produisent. Ainsi, la dernière phrase du premier chapitre de la première partie, sur les syndicats, affirme-t-elle que «la crise du syndicalisme français témoigne fondamentalement d'un grave déficit de légitimité démocratique» (p.47). Le chapitre sur le paritarisme se conclut sur une idée identique: "Autant de réalités qui minent la légitimité de dispositifs censés assurer la démocratie sociale» (p. 137). Celui sur la négociation collective évoque le manque d'autonomie et d'autorité de cette dernière en France (p. 214). Cette problématique est reformulée dans les dernières pages de l'ouvrage lorsque les auteurs posent la question: "Quelle démocratie sociale?» (pp. 255-256). Pour eux, il est clair que «le système de relations professionnelles français est à bout de souffle... Si l'on veut réellement envisager un avenir démocratique pour le monde du travail, alors il faut s'interroger sur les conditions d'émergence d'acteurs forts et légitimes.»

On peut regretter que l'ouvrage fasse une part trop réduite à la sociologie au profit des racines nord-américaines des relations professionnelles et des débats qu'elles suscitent. Durkheim n'apparaît ainsi que marginalement - à propos du rôle de l'État. Les théories sur la «société industrielle» (Friedmann, Aron...) sont de même absentes. Les débats européens autour du syndicalisme-plus politiques, dans la dynamique du marxisme - ne sont pas évoqués. On peut regretter également que les recherches autour des néocorporatismes - qui ont mobilisé des chercheurs européens comme nord-américains soient ignorées (3). Ils pourraient éclairer utilement la formation des syndicats et des spécificités nationales en Europe (pp. 29-33) et le chapitre sur «la confrontation sociale à la fracture sociale» (pp. 143-165).

Tel n'est pas cependant l'objectif de l'ouvrage, qui a le mérite de poser une bonne question: celle de la légitimité des acteurs du monde du travail, d'autant qu'elle ne concerne pas que la France.

(3) Pour une perspective sur ces théories, une bibliographie essentielle, et les débats à leur propos, $c f$. Lucio Baccaro (2002) "What is dead and what is alive in the theory of corporatism?" Working Paper, ILO, Geneva, DP/143/2002, 20 pages. Site: www.ilo.org/public/english/bureau/inst/download/dp14302.pdf 